\title{
Blood concentration of methylmercury in relation to food consumption
}

\author{
Riikka Airaksinen ${ }^{1, *}$, Anu W Turunen ${ }^{1}$, Panu Rantakokko ${ }^{1}$, Satu Männistö² \\ Terttu Vartiainen ${ }^{1}$ and Pia K Verkasalo ${ }^{1}$ \\ ${ }^{1}$ National Institute for Health and Welfare, Department of Environmental Health, PO Box 95, Fl-70701 Kuopio, \\ Finland: ${ }^{2}$ National Institute for Health and Welfare, Department of Chronic Disease Prevention, Helsinki, Finland
}

Submitted 27 October 2009: Accepted 13 April 2010: First published online 8 June 2010

\begin{abstract}
Objective: Dietary fish is the main source of methylmercury ( $\mathrm{MeHg}$ ) for man, and fish consumption has been used as a measure of MeHg exposure. However, other dietary sources of exposure exist and $\mathrm{MeHg}$ metabolism may also be modified by nutritional factors. The aim of the present study was to examine the association between blood $\mathrm{MeHg}$ concentration and consumption of different foods in a Finnish population with high fish consumption.

Design: Blood samples, a detailed FFQ and additional frequency data on fish consumption were collected. MeHg was analysed from whole blood by the isotope dilution method with high-resolution MS. The consumption of different foods was calculated by MeHg quartiles and tested for linear trend.

Setting: Finnish southern and south-western coast of the Baltic Sea.

Subjects: Two hundred and ninety-nine professional fishermen, their spouses and other family members.

Results: Mean (range) blood MeHg concentration was $4 \cdot 6(0 \cdot 21-22) \mu \mathrm{g} / \mathrm{l}$ among men and $2 \cdot 8(<0 \cdot 15-20) \mu \mathrm{g} / 1$ among women. Fish had the strongest positive association with $\mathrm{MeHg}$ ( $P$ for linear trend $<0 \cdot 001$ among both men and women). Among men, positive associations were also observed for fruit vegetables, wheat and wine. Among women, positive associations were observed for root vegetables, legumes, potato and game, but adjustment for fish consumption attenuated these trends.

Conclusions: The study shows that, besides fish, MeHg may have other dietary sources that should be taken into account in risk assessment studies. Due to the observed high blood $\mathrm{MeHg}$ concentration, a thorough exposure assessment among the general Finnish population is recommended.
\end{abstract}

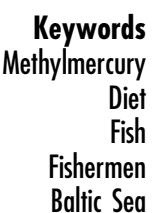

Along with the industrial revolution of the last century, several tragic poisoning episodes distinguished $\mathrm{Hg}$ as one of the most hazardous environmental contaminants. Due to its chemical nature, $\mathrm{Hg}$ is ubiquitous in the biosphere. In the environment, $\mathrm{Hg}$ exists in various chemical forms, the sources, transportation and fate of which are diverse $^{(1)}$. The most hazardous chemical form of $\mathrm{Hg}$ is methylmercury (MeHg), which has high lipid solubility and high affinity towards the sulfhydryl (-SH) groups of amino acids. Therefore, it accumulates efficiently in ecosystems. Food and especially large predatory fish at the top of the aquatic food chain are the main source of $\mathrm{MeHg}$ for man.

When considering low and chronic exposure to $\mathrm{MeHg}$, fetuses, infants and young children are the most vulnerable groups. In epidemiological studies, fetal exposure to moderate levels of $\mathrm{MeHg}$ has been associated with impaired neurological development, as deficits in cognitive, attention, motor and verbal tests ${ }^{(2-4)}$. In addition to the neurotoxic effects, high exposure to $\mathrm{Hg}$ has been associated with adverse cardiovascular effects among adults $^{(5)}$. To describe the level of exposure without recognized adverse effects during a lifetime, the US Environmental Protection Agency (EPA) has set a reference dose (RfD) of $0 \cdot 1 \mu \mathrm{g} / \mathrm{kg}$ body weight per $\mathrm{d}^{(6)}$, corresponding to a level of $3 \cdot 5 \mu \mathrm{g} \mathrm{MeHg} / 1$ in mother's blood ${ }^{(7)}$.

The health risk associated with exposure to $\mathrm{MeHg}$ has usually been assessed merely by measuring seafood consumption. However, the contribution of seafood to total MeHg burden varies between $20 \%$ and $85 \%$ in different countries, other sources being e.g. cereals and meat ${ }^{(8)}$. Furthermore, certain nutritional factors, such as Se or dietary fibre, may modify $\mathrm{MeHg}$ metabolism ${ }^{(9-11)}$. Ignoring exposure through other foods and not taking 
into account the modifying effects of nutrients may explain the somewhat inconsistent findings in epidemiological studies, i.e. that adverse health effects have been associated with prenatal exposure to $\mathrm{MeHg}$ in some studies but not all ${ }^{(12-14)}$.

In the present study, a population of Finnish professional fishermen and their family members was studied. Owing to very high fish consumption, this population is exposed to exceptionally high levels of fish-derived contaminants $^{(15)}$ and offers a unique resource to assess $\mathrm{MeHg}$ exposure. The objective was to assess the association between blood $\mathrm{MeHg}$ and the consumption of different foods among the fishermen and their family members and to identify potential dietary sources of MeHg.

\section{Materials and methods}

\section{Study population, sampling and dietary assessment}

The Fishermen Study Cohort consisted of all Finnish professional fishermen as well as their spouses and other family members living at the southern and south-western Baltic Sea coast of Finland ${ }^{(15)}$. From this cohort, a subsample of 309 participants attended a health examination study (the Fishermen Sub-study) between August 2004 and May 2005.

The health examination included blood sampling and a validated $^{(16,17)}$, self-administered, 128-item FFQ, including ten fish items. The FFQ was designed to cover the whole diet over the preceding 12 months. The participants were asked to indicate the frequency of consumption of each food item with nine frequency categories ranging from 'never' to 'six or more times per day' (for more information, see Appendix in Turunen et al. $\left.{ }^{(18)}\right)$. Portion sizes were specified using commonly used units (e.g. glass, slice or serving). The FFQ data were processed using the national Fineli ${ }^{\circledR}$ Finnish Food Composition Database (National Institute for Health and Welfare) ${ }^{(19)}$ and the food consumption frequencies were converted into amounts of ingredients eaten $(\mathrm{g} / \mathrm{d})$.

In addition to the FFQ on whole diet, the participants completed a separate health questionnaire which included more detailed questions on the consumption of different fish species (servings/month). In the health questionnaire, the participants were asked to indicate the frequency of use of frozen fish (e.g. coalfish, cod, redfish, fish sticks); canned ocean fish (e.g. tuna, sardine, herring, mackerel); rainbow trout (e.g. fresh, frozen, canned); Baltic herring (e.g. fresh, frozen, canned); predatory fish from inland waters (e.g. pike, perch, burbot, pike-perch); vendace; other fish from inland waters (e.g. whitefish, bream, roach); Baltic salmon and trout; other Baltic fish; and other ocean fish (e.g. smoked mackerel, Norwegian salmon). There were six possible frequency categories, ranging from 'never' to 'almost every day', and portion sizes were not specified.
Complete data from the blood sampling, FFQ on whole diet and the separate health questionnaire were obtained from 301 participants. The majority (80\%) of the male participants were professional fishermen and most (68\%) of the female participants were fishermen's wives. Blood $\mathrm{MeHg}$ concentrations were analysed at the National Institute for Health and Welfare, Chemical Exposure Unit, which is an accredited testing laboratory (Code T077, EN ISO/IEC 17025).

\section{Sample preparation and quantification using isotope-dilution GC/bigh-resolution MS}

Sodium citrate was used as an anticoagulant and the blood samples were stored at $-70^{\circ} \mathrm{C}$ prior to analysis. An isotope dilution method was developed for the present study in order to accurately measure low concentrations of $\mathrm{MeHg}$ from the blood samples and the method is therefore described here in detail. Methylmercury chloride of natural isotopic abundances of $\mathrm{Hg}$ and an isotopically enriched (to $96 \cdot 41 \%$ in the $\mathrm{CH}_{3}{ }^{200} \mathrm{HgCl}$ isotopomer) spike solution of methylmercury chloride in methanol were purchased from Applied Isotope Technologies Inc. (Sunnyvale, CA, USA). The method used for the quantification of $\mathrm{MeHg}$ from whole blood sample was modified from the method of Baxter et al. ${ }^{(20)}$. In brief, $5 \mathrm{ng}$ of enriched $\mathrm{CH}_{3}{ }^{200} \mathrm{HgCl}$ spike in $165 \mu \mathrm{l}$ of methanol was added to $1 \mathrm{ml}$ blood sample in a $12 \mathrm{ml}$ screw-capped test-tube and the sample shaken for $4 \mathrm{~h}$ in order to allow the enriched methylmercury to equilibrate. Three $\mathrm{ml}$ of $1.4 \mathrm{~m}-\mathrm{NaBr}$ in $0.9 \mathrm{M}-\mathrm{H}_{2} \mathrm{SO}_{4}$ and $1 \mathrm{ml}$ of $1 \mathrm{M}^{-} \mathrm{CuSO}_{4}$ were added to the sample to release the $\mathrm{CH}_{3} \mathrm{Hg}^{+}$cation from protein-binding sites and to form a stable methylmercury-bromide complex. The methylmercury-bromide complex was extracted into $6 \mathrm{ml}$ of hexane. The hexane phase was separated and $1 \mathrm{ml}$ of $0 \cdot 1 \mathrm{M}-\mathrm{NaHCO}_{3}$ buffer solution and $2 \mathrm{ml}$ of sodium tetraphenylborate derivatization reagent $(0.25 \% \mathrm{w} / \mathrm{v}$ in water) were added to form the volatile phenyl derivative of methylmercury $\left(\mathrm{CH}_{3} \mathrm{HgC}_{6} \mathrm{H}_{5}\right)$. The aqueous phase was discarded and hexane was changed to $0.5 \mathrm{ml}$ of toluene as the final solvent. The phenyl derivative of methylmercury was analysed with an HP 6890 gas chromatograph (Agilent Technologies, Inc., Wilmington, DE, USA) connected to an Autospec Ultima high-resolution mass spectrometer (HRMS; Waters, Manchester, UK) operating in the selected-ion monitoring mode using a resolution of 8000 . The column used was a DB-5MS capillary column $(12 \mathrm{~m}, 0.25 \mathrm{~mm}$ internal diameter, $0 \cdot 25 \mu \mathrm{m}$ film; Agilent Technologies, Inc.). The areas of molecular ions $\mathrm{m} / z 292 \cdot 0311$ and $294 \cdot 0334$ were monitored. Theoretical relative abundances of these ions in the HRMS spectra for both natural and enriched spike solution were calculated using the isotope pattern calculator ${ }^{(21)}$. From the theoretical and observed ion ratios and the mass of enriched spike added to the samples, the original concentration $\mathrm{MeHg}$ in the blood samples was calculated using the principles and equations presented by Yang et al. ${ }^{(22)}$. 


\section{Quality control and assurance}

To avoid contamination, all glassware was rinsed with $0 \cdot 01 \mathrm{~m}-\mathrm{HCl}-\mathrm{EtOH}$ solution, soaked overnight in $1 \mathrm{~m}-\mathrm{HNO}_{3}$ and heated at $500^{\circ} \mathrm{C}$ for $8 \mathrm{~h}$. With each series of samples, a blank sample and two internal control samples (bovine and human blood with known additions of $\mathrm{MeHg}$ ) were analysed. The limit of quantification was $0 \cdot 15 \mu \mathrm{g} / \mathrm{l}$. For bovine blood ( $n$ 13), percentage recovery was 108 and the percentage relative standard deviation (\%RSD) was $7 \cdot 5$. For human blood ( $n 13)$, percentage recovery was 94 and the \%RSD was $4 \cdot 2$. During each GC-HRMS sample series, an ion intensity ratio control solution ( $50 \mathrm{ng}$ natural abundance phenylated methylmercury/ml solution) was measured three times to correct for the mass bias of the HRMS instrument. Two participants were excluded from the statistical analyses since their blood $\mathrm{MeHg}$ concentrations ( 48 and $60 \mu \mathrm{g} / \mathrm{l}$ ) were above the measurement range of the analytical method.

\section{Statistical analyses}

In order to determine the potential dietary sources of $\mathrm{MeHg}$, age- and energy-adjusted consumption of foods by $\mathrm{MeHg}$ quartiles were calculated and tested for linear trend across quartiles. Since fish consumption was strongly associated with the consumption of certain other foods (data not shown) and very significantly associated with blood MeHg, the consumption of other foods was further adjusted for fish consumption. To study the foods with very low consumption frequency more precisely, the statistical analysis was repeated only among those participants who reported to have consumed each food at least once during the last 12 months, i.e. non-users were excluded (data not shown). A $P$ value of 0.05 was selected as the threshold of statistical significance.

\section{Results}

The final number of participants in the present study was 299, of whom 137 were men and 162 were women. Mean blood MeHg concentration was $4.6 \mu \mathrm{g} / \mathrm{l}$ among men and $2 \cdot 8 \mu \mathrm{g} / \mathrm{l}$ among women (Table 1 ). In total, $32 \%$ of the participants exceeded the US EPA RfD-derived MeHg concentration of $3.5 \mu \mathrm{g} / \mathrm{l}$. The blood $\mathrm{MeHg}$ concentration as well as the percentage of the participants exceeding $3.5 \mu \mathrm{g} / 1$ increased with increasing age and fish consumption. The mean (range) blood $\mathrm{MeHg}$ concentrations by MeHg quartiles among men were $1 \cdot 1(0 \cdot 21-1 \cdot 9) \mu \mathrm{g} / \mathrm{l}$, $2 \cdot 7(2 \cdot 0-3 \cdot 3) \mu \mathrm{g} / 1,4 \cdot 5(3 \cdot 4-6 \cdot 4) \mu \mathrm{g} / 1$ and $10(6 \cdot 5-22) \mu \mathrm{g} /$ 1. Among women, the respective concentrations were $0 \cdot 71(0-1 \cdot 2) \mu \mathrm{g} / 1,1 \cdot 7(1 \cdot 3-2 \cdot 0) \mu \mathrm{g} / 1,2 \cdot 8(2 \cdot 1-3 \cdot 3) \mu \mathrm{g} / 1$ and $6 \cdot 0(3 \cdot 4-20) \mu \mathrm{g} / \mathrm{l}$.

Fish consumption had the strongest association with blood MeHg (FFQ on whole diet): those who had the highest blood $\mathrm{MeHg}$ concentration had the highest fish consumption among both men and women $(P<0 \cdot 001$; Tables 2 and 3). With regard to fish species (frequency questions on fish consumption), the strongest associations with MeHg were observed for Baltic fish: herring $(P=0.51$ among men and 0.002 among women), salmon

Table 1 Blood MeHg concentration (unadjusted, $\mu \mathrm{g} / \mathrm{l}$ ) according to sex, age and fish consumption ${ }^{*}, \uparrow$ and the percentage of participants exceeding the US EPA RfDł: Fishermen Sub-study, Finland, August 2004-May 2005

\begin{tabular}{|c|c|c|c|c|c|c|c|}
\hline & \multirow[b]{2}{*}{$n$} & \multicolumn{5}{|c|}{ Blood $\mathrm{MeHg}(\mu \mathrm{g} / \mathrm{l})$} & \multirow[b]{2}{*}{$\%>\operatorname{RfD} \ddagger$} \\
\hline & & Mean & Median & Min. & Max. & $\mathrm{P} 90$ & \\
\hline Total & 299 & $3 \cdot 6$ & $2 \cdot 7$ & $<0 \cdot 15$ & 22 & $8 \cdot 0$ & 32 \\
\hline \multicolumn{8}{|l|}{ Sex } \\
\hline Men & 137 & $4 \cdot 6$ & $3 \cdot 4$ & 0.21 & 22 & $9 \cdot 1$ & 45 \\
\hline Women & 162 & $2 \cdot 8$ & $2 \cdot 1$ & $<0 \cdot 15$ & 20 & $5 \cdot 7$ & 21 \\
\hline \multicolumn{8}{|l|}{ Age (years) } \\
\hline $21-39$ & 52 & 1.9 & $1 \cdot 4$ & $<0.15$ & 15 & $3 \cdot 5$ & $9 \cdot 6$ \\
\hline $40-49$ & 64 & $3 \cdot 8$ & $2 \cdot 5$ & $<0 \cdot 15$ & 22 & $8 \cdot 8$ & 34 \\
\hline 50-59 & 103 & $3 \cdot 6$ & $2 \cdot 9$ & 0.42 & 19 & $6 \cdot 7$ & 33 \\
\hline $60-75$ & 80 & $4 \cdot 5$ & $3 \cdot 4$ & $<0 \cdot 15$ & 20 & 8.9 & 43 \\
\hline \multicolumn{8}{|c|}{ Fish consumption $(\mathrm{g} / \mathrm{d})^{*}$} \\
\hline$<40$ & 78 & $2 \cdot 1$ & $1 \cdot 3$ & $<0 \cdot 15$ & 22 & $3 \cdot 6$ & 14 \\
\hline $40-50$ & 47 & $3 \cdot 2$ & $2 \cdot 2$ & 0.58 & 19 & $6 \cdot 8$ & 21 \\
\hline $50-70$ & 57 & $3 \cdot \overline{7}$ & $2 \cdot 8$ & 0.58 & 11 & $7 \cdot 7$ & 35 \\
\hline $70-100$ & 61 & $3 \cdot 6$ & $3 \cdot 2$ & 0.93 & 15 & $5 \cdot 7$ & 36 \\
\hline$>100$ & 56 & $5 \cdot 9$ & 4.9 & 0.48 & 20 & 10 & 57 \\
\hline \multicolumn{8}{|c|}{ Fish consumption (servings/month)† } \\
\hline$<4$ & 26 & $2 \cdot 0$ & 0.89 & $<0 \cdot 15$ & 22 & $3 \cdot 2$ & $7 \cdot 7$ \\
\hline $4-7 \cdot 9$ & 77 & $2 \cdot 6$ & $1 \cdot 8$ & $<0 \cdot 15$ & 10 & $6 \cdot 2$ & 22 \\
\hline $8-11 \cdot 9$ & 68 & 3.6 & $2 \cdot 8$ & $<0.15$ & 19 & $7 \cdot 6$ & 32 \\
\hline $12-15.9$ & 49 & $3 \cdot 1$ & $2 \cdot 5$ & 0.69 & 9.9 & $5 \cdot 9$ & 22 \\
\hline$\geq 16$ & 79 & $5 \cdot 4$ & $3 \cdot 7$ & 0.48 & 20 & 10 & 54 \\
\hline
\end{tabular}

MeHg, methylmercury; EPA, Environmental Protection Agency; RfD, reference dose.

*Based on the FFQ.

tBased on the additional health questionnaire frequencies on consumption of specified fish species.

$\ddagger 3.5 \mu \mathrm{g} / \mathrm{l}$, derived from US EPA RfD of $0.1 \mu \mathrm{g} / \mathrm{kg}$ body weight per $\mathrm{d}$. 
or trout $(P=0.04$ and 0.84$)$ and other Baltic fish $(P<0.001$ and $0 \cdot 01$, including e.g. Baltic pike, perch, burbot, pike-perch, whitefish and bream; Tables 4 and 5). Among men, other ocean fish $(P=0 \cdot 04$, including e.g. smoked mackerel and Norwegian salmon) and shellfish $(P=0 \cdot 01)$ also had a statistically significant positive trend across quartiles. Among women, freshwater predatory fish $(P=0 \cdot 007)$ had a strong positive trend across $\mathrm{MeHg}$ quartiles, but no trend was observed for either other ocean fish or shellfish.

Total vegetable consumption (FFQ on whole diet, Tables 2 and 3) was positively associated with blood MeHg $(P=0.03$ among men and 0.09 among women). Among men, the association was largely due to fruit vegetables, which had a statistically significant positive trend across quartiles $(P=0 \cdot 002)$ also after adjustment for fish consumption $(P<0 \cdot 001)$. Fruit vegetables include the most popular Finnish salad vegetables: tomato, sweet pepper, cucumber and courgette. Among women, no trend across quartiles was observed for fruit vegetables. Instead, a positive trend was observed for root vegetables $(P=0 \cdot 004)$, legumes $(P=0 \cdot 03)$ and mushrooms $(P=0 \cdot 07)$. However, the trend for root vegetables attenuated to borderline significant $(P=0 \cdot 05)$ and the trends for legumes and mushrooms did not remain statistically significant after adjustment for fish consumption $(P=0 \cdot 16$ and $0 \cdot 29$, respectively).

Potato had a borderline significant positive trend across quartiles among women $(P=0 \cdot 05)$, but adjustment for fish consumption attenuated the trend to non-significant $(P=0 \cdot 13$; Table 3$)$. Among men, the consumption of potato increased up to the third quartile after declining, and the linear trend test was statistically non-significant (Table 2).

Wheat $(P=0.04)$ and rye $(P=0 \cdot 15)$ had a positive trend across MeHg quartiles among men (Table 2), also after adjustment for fish consumption $(P=0.02$ for wheat and $P=0.05$ for rye). Among women, no trend across quartiles was observed for cereals (Table 3).

Total meat consumption did not differ across $\mathrm{MeHg}$ quartiles (Tables 2 and 3). Among women, game consumption $(P=0 \cdot 03)$ had a statistically significant positive trend, but the trend was attenuated by adjustment for fish consumption $(P=0 \cdot 10$; Table 3$)$. Among men, the respective trends were not statistically significant $(P=0.98$ and 0.70 ; Table 2$)$, but when non-users were excluded, a statistically significant positive trend was observed for the consumption of offal $(P=0.03$ with adjustment for age and energy, $P=0.05$ with further adjustment for fish consumption). For other foods, excluding the non-users did not affect the results among men or women (data not shown).

Alcoholic beverages $(P=0 \cdot 03)$ and especially wine $(P=0.02)$ had a statistically significant positive trend across MeHg quartiles among men (Table 2). Further adjustment for fish consumption did not change the associations $(P=0 \cdot 03$ and $0 \cdot 01$, respectively). There was a similar, but statistically non-significant trend among women (Table 3). It should be noted that the units are given in grams of alcoholic beverages, not in grams of ethanol.

\section{Discussion}

The average blood MeHg concentration was high $(<0 \cdot 15-22 \mu \mathrm{g} / 1)$ in the study population. Among men, the concentration was twice as high as among women. The $\mathrm{MeHg}$ concentration was in line with the participants' high fish consumption. In the present study, mean fish and shellfish consumption was $76 \mathrm{~g} / \mathrm{d}$ among men and $66 \mathrm{~g} / \mathrm{d}$ among women, while the average Finnish fish consumption is approximately 48 and $42 \mathrm{~g} / \mathrm{d}$ among men and women, respectively ${ }^{(23)}$. To our knowledge, $\mathrm{MeHg}$ has not previously been measured from blood in Finland. However, in a previous Finnish study in a population with fish consumption closer to the national average, hair concentration of $\mathrm{Hg}$ was lower than the blood concentration of $\mathrm{MeHg}$ found in the present study, when a hair-to-blood ratio of 250:1 was applied $^{(5)}$.

Worldwide, varied $\mathrm{Hg}$ concentrations have been measured from whole blood among populations with equally high fish consumption as in the present study. For example, among Swedish fishermen, the median concentration of $\mathrm{MeHg}$ was $4 \mu \mathrm{g} / \mathrm{l}(1 \cdot 6-9 \cdot 0 \mu \mathrm{g} / \mathrm{l})^{(24)}$; among Canadian anglers and sport-fish-eaters, the concentrations of $\mathrm{MeHg}$ ranged between 0 and $15 \cdot 8 \mu \mathrm{g} / \mathrm{l}^{(25)}$; and among Taiwanese pregnant women with high fish consumption, the total $\mathrm{Hg}$ median was $8 \cdot 3 \mu \mathrm{g} / 1(4 \cdot 4-21 \mu \mathrm{g} / \mathrm{l})^{(26)}$. There is high inter-individual variation in the total $\mathrm{Hg}$-to-MeHg ratio in whole blood ${ }^{(27)}$. The proportion of $\mathrm{MeHg}$ from total $\mathrm{Hg}$ in blood may sometimes vary as much as 6-100\% ${ }^{(20)}$, which complicates the comparisons between these studies. However, the inconsistent concentrations observed in the aforementioned studies may also be partly explained by analytical and methodological differences, as well as differences in the type and contamination of fish consumed, other dietary sources of $\mathrm{Hg}$ and sources and intake of $\mathrm{Hg}$ metabolism modifiers. For example, dietary fibre may decrease $\mathrm{MeHg}$ absorption ${ }^{(28)}$ whereas milk may promote it ${ }^{(29)}$.

In Finland, the general population is advised by the National Nutrition Council to eat fish of varying species at least twice weekly. In the present study, $24 \%$ of the participants consuming fish twice weekly at the most exceeded the blood $\mathrm{MeHg}$ level of $3.5 \mu \mathrm{g} / \mathrm{l}$, derived from the US EPA's RfD. Among the participants consuming fish more than twice weekly, the respective proportion was $42 \%$. The threshold level of $3.5 \mu \mathrm{g} / 1$ has been calculated for mother's blood, with the aim of safeguarding the fetus. Therefore, exceeding this limit may be irrelevant for the adult population. However, the high proportion of 
Table 2 Adjusted means for consumption of selected foods (FFQ, g/d) across blood MeHg quartiles for men: Fishermen Sub-study, Finland, August 2004-May 2005

\begin{tabular}{|c|c|c|c|c|c|c|c|c|c|c|c|c|c|c|}
\hline \multirow[b]{3}{*}{ Food groups } & \multicolumn{7}{|c|}{ Adjusted for age and energy intake } & \multicolumn{7}{|c|}{ Adjusted for age, energy intake and fish consumption } \\
\hline & \multicolumn{2}{|c|}{$\begin{array}{l}\text { Total consumption }(\mathrm{g} / \mathrm{d}) \\
(n 137)\end{array}$} & \multicolumn{4}{|c|}{$\begin{array}{l}\text { Mean consumption by } \\
\text { MeHg quartiles (g/d) }\end{array}$} & \multirow{2}{*}{$\begin{array}{l}P \text { for } \\
\text { linear } \\
\text { trend }\end{array}$} & \multicolumn{2}{|c|}{$\begin{array}{l}\text { Total consumption }(\mathrm{g} / \mathrm{d}) \\
(n \text { 137) }\end{array}$} & \multicolumn{4}{|c|}{$\begin{array}{l}\text { Mean consumption by } \\
\text { MeHg quartiles }(\mathrm{g} / \mathrm{d})\end{array}$} & \multirow{2}{*}{$\begin{array}{l}P \text { for } \\
\text { linear } \\
\text { trend }\end{array}$} \\
\hline & Mean & $95 \% \mathrm{Cl}$ & Q1 (n 34) & Q2 (n 34) & Q3 (n 35) & Q4 (n 34) & & Mean & $95 \% \mathrm{Cl}$ & Q1 (n 34) & Q2 (n 34) & Q3 (n 35) & Q4 (n 34) & \\
\hline Fruits & 223 & 190,257 & 261 & 244 & 202 & 244 & $0 \cdot 80$ & 225 & 191,259 & 247 & 242 & 205 & 259 & 0.73 \\
\hline Vegetables & 207 & 182,233 & 217 & 211 & 193 & 268 & 0.03 & 206 & 181,232 & 212 & 210 & 194 & 273 & 0.02 \\
\hline Fruit vegetables & 97 & 80,114 & 91 & 95 & 89 & 145 & 0.002 & 97 & 80,114 & 86 & 94 & 90 & 150 & $<0.001$ \\
\hline Root vegetables & 35 & 29,41 & 42 & 37 & 33 & 36 & 0.41 & 34 & 28,41 & 44 & 37 & 33 & 34 & 0.20 \\
\hline Mushrooms & $4 \cdot 8$ & $4 \cdot 0,5 \cdot 7$ & $4 \cdot 7$ & $4 \cdot 6$ & $4 \cdot 6$ & $6 \cdot 2$ & $0 \cdot 12$ & $4 \cdot 8$ & $4 \cdot 0,5 \cdot 6$ & 4.5 & $4 \cdot 6$ & $4 \cdot 6$ & $6 \cdot 4$ & 0.09 \\
\hline Legumes & 12 & 10,14 & 13 & 12 & 12 & 12 & 0.62 & 12 & 10,13 & 13 & 12 & 12 & 12 & 0.74 \\
\hline Other vegetables* & 58 & 52,65 & 66 & 63 & 54 & 69 & 0.52 & 58 & 51,65 & 65 & 63 & 54 & 71 & 0.37 \\
\hline Potato & 191 & 175,208 & 195 & 217 & 237 & 176 & 0.25 & 189 & 173,205 & 209 & 219 & 234 & 162 & 0.04 \\
\hline Cereals & 156 & 148,164 & 156 & 168 & 170 & 170 & 0.39 & 157 & 149,164 & 149 & 167 & 172 & 177 & $0 \cdot 06$ \\
\hline Rye & 41 & 36,46 & 39 & 43 & 41 & 51 & $0 \cdot 15$ & 41 & 36,46 & 36 & 43 & 42 & 53 & 0.05 \\
\hline Wheat & 80 & 74,85 & 72 & 85 & 95 & 91 & 0.04 & 80 & 75,85 & 70 & 85 & 95 & 93 & 0.02 \\
\hline Other cerealst & 35 & 31,40 & 45 & 40 & 34 & 29 & 0.002 & 36 & 31,40 & 43 & 39 & 35 & 31 & 0.03 \\
\hline Milk & 501 & 452,551 & 697 & 470 & 507 & 461 & 0.01 & 510 & 461,558 & 667 & 465 & 512 & 490 & $0 \cdot 10$ \\
\hline Fat & 41 & 39,44 & 42 & 46 & 42 & 47 & 0.19 & 41 & 39,43 & 43 & 46 & 42 & 46 & 0.50 \\
\hline Eggs & 30 & 26,33 & 31 & 33 & 31 & 32 & 0.95 & 30 & 26,33 & 32 & 34 & 31 & 31 & 0.66 \\
\hline Fish and shellfish & 76 & 68,84 & 49 & 76 & 87 & 113 & $<0.001$ & & & & & & & \\
\hline Fish & 64 & 56,71 & 42 & 64 & 71 & 96 & $<0.001$ & & & & & & & \\
\hline Fish products & 10 & $8 \cdot 8,12$ & $6 \cdot 4$ & 10 & 14 & 14 & 0.008 & & & & & & & \\
\hline Shellfish & $1 \cdot 7$ & $1 \cdot 2,2 \cdot 3$ & 0.54 & 1.9 & $1 \cdot 8$ & $2 \cdot 7$ & 0.03 & & & & & & & \\
\hline Meat & 168 & 156,180 & 179 & 175 & 173 & 176 & 0.90 & 168 & 156,180 & 183 & 176 & 173 & 172 & 0.64 \\
\hline Game & $7 \cdot 3$ & $4 \cdot 7,10$ & $8 \cdot 3$ & $6 \cdot 8$ & $5 \cdot 8$ & $7 \cdot 6$ & 0.98 & $7 \cdot 3$ & $4 \cdot 7,10$ & $7 \cdot 4$ & $6 \cdot 6$ & 5.9 & $8 \cdot 5$ & 0.70 \\
\hline Offal & $4 \cdot 8$ & $3 \cdot 7,6 \cdot 0$ & $2 \cdot 3$ & $6 \cdot 6$ & $6 \cdot 4$ & $5 \cdot 6$ & 0.35 & $4 \cdot 7$ & $3 \cdot 6,5 \cdot 9$ & $2 \cdot 8$ & $6 \cdot 7$ & $6 \cdot 3$ & $5 \cdot 1$ & 0.64 \\
\hline Other meat & 156 & 145,167 & 169 & 162 & 161 & 162 & 0.81 & 156 & 145,168 & 173 & 162 & 160 & 159 & 0.53 \\
\hline Alcoholic beverages & 181 & 155,208 & 126 & 171 & 207 & 239 & 0.03 & 180 & 154,207 & 122 & 170 & 207 & 243 & 0.03 \\
\hline Wines & 30 & 15,45 & $8 \cdot 7$ & 26 & 16 & 80 & 0.02 & 29 & 14,45 & $4 \cdot 4$ & 25 & 17 & 85 & 0.01 \\
\hline Other alcoholic beverages $\S$ & 152 & 131,172 & 117 & 145 & 191 & 159 & 0.34 & 151 & 130,172 & 118 & 145 & 191 & 159 & 0.39 \\
\hline
\end{tabular}

MeHg, methylmercury.

*Leaf vegetables, cabbage, onions, nuts, seeds and soya products.

ind miscellaneous cereals.

sBeers, spirits and other miscellaneous alcoholic beverages. products. 
Table 3 Adjusted means for consumption of selected foods (FFQ, g/d) across blood MeHg quartiles for women: Fishermen Sub-study, Finland, August 2004-May 2005

\begin{tabular}{|c|c|c|c|c|c|c|c|c|c|c|c|c|c|c|}
\hline \multirow[b]{3}{*}{ Food groups } & \multicolumn{7}{|c|}{ Adjusted for age and energy intake } & \multicolumn{7}{|c|}{ Adjusted for age, energy intake and fish consumption } \\
\hline & \multicolumn{2}{|c|}{$\begin{array}{c}\text { Total consumption }(\mathrm{g} / \mathrm{d}) \\
(n \text { 162) }\end{array}$} & \multicolumn{4}{|c|}{$\begin{array}{l}\text { Mean consumption by } \\
\text { MeHg quartiles }(g / d)\end{array}$} & \multirow{2}{*}{$\begin{array}{l}P \text { for } \\
\text { linear } \\
\text { trend }\end{array}$} & \multicolumn{2}{|c|}{$\begin{array}{l}\text { Total consumption }(\mathrm{g} / \mathrm{d}) \\
(\text { ( } 162)\end{array}$} & \multicolumn{4}{|c|}{$\begin{array}{l}\text { Mean consumption by } \\
\text { MeHg quartiles }(g / d)\end{array}$} & \multirow{2}{*}{$\begin{array}{l}P \text { for } \\
\text { linea } \\
\text { trend }\end{array}$} \\
\hline & Mean & $95 \% \mathrm{Cl}$ & Q1 ( $n$ 42) & Q2 (n 39) & Q3 ( $n$ 40) & Q4 (n 41) & & Mean & $95 \% \mathrm{Cl}$ & Q1 ( $n$ 42) & Q2 (n 39) & Q3 (n 40) & Q4 (n 41) & \\
\hline Fruits & 328 & 297, 359 & 337 & 288 & 319 & 317 & 0.94 & 326 & 295,357 & 343 & 288 & 316 & 314 & 0.84 \\
\hline Vegetables & 331 & 307,354 & 291 & 325 & 292 & 365 & 0.09 & 332 & 308,355 & 306 & 326 & 284 & 357 & 0.25 \\
\hline Fruit vegetables & 167 & 151,183 & 145 & 181 & 147 & 171 & 0.58 & 167 & 151,183 & 150 & 181 & 145 & 169 & 0.75 \\
\hline Root vegetables & 55 & 49,60 & 45 & 47 & 48 & 72 & 0.004 & 55 & 49,61 & 51 & 47 & 45 & 68 & 0.05 \\
\hline Mushrooms & $5 \cdot 7$ & $4 \cdot 9,6 \cdot 4$ & $4 \cdot 6$ & 4.9 & 5.9 & $6 \cdot 7$ & 0.07 & $5 \cdot 7$ & $4 \cdot 9,6 \cdot 5$ & $5 \cdot 3$ & 4.9 & $5 \cdot 6$ & $6 \cdot 3$ & 0.29 \\
\hline Legumes & 14 & 12,15 & 11 & 11 & 14 & 16 & 0.03 & 14 & 12,15 & 12 & 11 & 13 & 16 & $0 \cdot 16$ \\
\hline Other vegetables* & 90 & 84,96 & 86 & 82 & 77 & 99 & $0 \cdot 12$ & 90 & 84,96 & 88 & 82 & 76 & 98 & 0.22 \\
\hline Potato & 174 & 159,189 & 143 & 157 & 166 & 180 & 0.05 & 176 & 161,191 & 148 & 157 & 163 & 177 & $0 \cdot 13$ \\
\hline Cereals & 163 & 156,170 & 153 & 150 & 155 & 161 & 0.31 & 163 & 156,170 & 155 & 150 & 154 & 160 & 0.47 \\
\hline Rye & 41 & 36,46 & 37 & 38 & 35 & 46 & $0 \cdot 18$ & 41 & 36,46 & 40 & 38 & 33 & 44 & 0.39 \\
\hline Wheat & 76 & 71,81 & 70 & 72 & 75 & 66 & 0.43 & 76 & 71,81 & 67 & 72 & 76 & 68 & 0.90 \\
\hline Other cerealst & 46 & 42,50 & 45 & 40 & 45 & 49 & 0.32 & 46 & 42,50 & 48 & 40 & 44 & 47 & 0.74 \\
\hline Milk & 554 & 508,600 & 549 & 549 & 568 & 443 & 0.07 & 547 & 502,591 & 506 & 549 & 591 & 465 & 0.36 \\
\hline Fat & 43 & 41,45 & 37 & 45 & 39 & 40 & 0.90 & 43 & 41,45 & 39 & 45 & 38 & 39 & 0.57 \\
\hline Eggs & 27 & 24,30 & 28 & 26 & 24 & 23 & 0.31 & 27 & 24,30 & 25 & 26 & 26 & 24 & 0.74 \\
\hline Fish and shellfish & 66 & 58,73 & 35 & 62 & 75 & 75 & $<0.001$ & & & & & & & \\
\hline Fish & 55 & 48,62 & 29 & 51 & 63 & 64 & $<0.001$ & & & & & & & \\
\hline Fish products & $8 \cdot 4$ & $6 \cdot 9,10$ & $4 \cdot 7$ & 8.6 & 10 & $8 \cdot 4$ & 0.18 & & & & & & & \\
\hline Shellfish & $2 \cdot 2$ & $1 \cdot 7,2 \cdot 7$ & $1 \cdot 8$ & 2.5 & $2 \cdot 0$ & $2 \cdot 3$ & $0 \cdot 70$ & & & & & & & \\
\hline Meat & 152 & 141,163 & 154 & 150 & 137 & 141 & $0 \cdot 40$ & 152 & 141,163 & 146 & 150 & 141 & 145 & 0.89 \\
\hline Game & $6 \cdot 3$ & $4 \cdot 0,8 \cdot 7$ & $4 \cdot 8$ & $4 \cdot 8$ & $4 \cdot 8$ & 11 & 0.03 & $6 \cdot 3$ & $4 \cdot 0,8 \cdot 6$ & $6 \cdot 0$ & $4 \cdot 8$ & $4 \cdot 2$ & 11 & $0 \cdot 10$ \\
\hline Offal & $4 \cdot 3$ & $3 \cdot 3,5 \cdot 3$ & $3 \cdot 2$ & $3 \cdot 4$ & $4 \cdot 6$ & $4 \cdot 8$ & $0 \cdot 11$ & $4 \cdot 4$ & $3 \cdot 4,5 \cdot 4$ & $3 \cdot 4$ & $3 \cdot 4$ & $4 \cdot \overline{5}$ & $4 \cdot 7$ & 0.20 \\
\hline Other meatł & 141 & 131,152 & 146 & 142 & 127 & 125 & $0 \cdot 13$ & 141 & 131,152 & 137 & 142 & 132 & 130 & 0.53 \\
\hline Alcoholic beverages & 70 & 46,94 & 39 & 66 & 86 & 74 & 0.22 & 71 & 46,95 & 33 & 66 & 90 & 77 & 0.13 \\
\hline Wines & 19 & $4 \cdot 8,32$ & 13 & 18 & 17 & 17 & 0.59 & 19 & $5 \cdot 0,33$ & 12 & 18 & 17 & 18 & 0.49 \\
\hline Other alcoholic beverages $\S$ & 51 & 32,70 & 26 & 48 & 70 & 56 & 0.21 & 52 & 33,71 & 21 & 48 & 72 & 59 & 0.13 \\
\hline
\end{tabular}

MeHg, methylmercury.

Leaf vegetables, cabbage, onions, nuts, seeds and soya products.

tOats, barley, rice, starch and other miscellaneous cereals.

‡Beef, pork, mutton, poultry and other miscellaneous meat products.

$\S$ Beers, spirits and other miscellaneous alcoholic beverages. 
Table 4 Adjusted means for consumption of specified fish species (separate health questionnaire, servings/month) across blood MeHg quartiles for men: Fishermen Sub-study, Finland, August 2004-May 2005

\begin{tabular}{|c|c|c|c|c|c|c|c|}
\hline \multirow[b]{3}{*}{ Food groups } & \multicolumn{7}{|c|}{ Adjusted for age and energy intake } \\
\hline & \multicolumn{2}{|c|}{$\begin{array}{l}\text { Total consumption } \\
\text { (servings/month) }(n 137)\end{array}$} & \multicolumn{4}{|c|}{$\begin{array}{l}\text { Mean consumption by MeHg quartiles } \\
\text { (servings/month) }\end{array}$} & \multirow{2}{*}{$\begin{array}{l}P \text { for linear } \\
\text { trend }\end{array}$} \\
\hline & Mean & $95 \% \mathrm{Cl}$ & Q1 (n 34) & Q2 (n 34) & Q3 (n 35) & Q4 (n 34) & \\
\hline Fish in total & 13 & 12,15 & $8 \cdot 4$ & 13 & 15 & 18 & $<0.001$ \\
\hline Baltic ocean fish & $7 \cdot 1$ & $6 \cdot 0,8 \cdot 1$ & $3 \cdot 2$ & $7 \cdot 3$ & $8 \cdot 0$ & 11 & $<0.001$ \\
\hline Baltic herring & $2 \cdot 4$ & $2 \cdot 0,2 \cdot 8$ & $1 \cdot \overline{6}$ & $3 \cdot 0$ & $2 \cdot 9$ & $2 \cdot 5$ & 0.51 \\
\hline Baltic salmon or trout & $1 \cdot 1$ & $0 \cdot 82,1 \cdot 4$ & $0 \cdot 82$ & $1 \cdot 0$ & $1 \cdot 0$ & $1 \cdot 6$ & 0.04 \\
\hline Other Baltic fish & $3 \cdot 6$ & $2 \cdot 8,4 \cdot 4$ & $0 \cdot 81$ & $3 \cdot 3$ & $4 \cdot 1$ & $6 \cdot 5$ & $<0.001$ \\
\hline Other ocean fish & $2 \cdot 5$ & $1 \cdot 9,3 \cdot 1$ & $1 \cdot 8$ & $2 \cdot 2$ & $2 \cdot 5$ & $3 \cdot 5$ & 0.04 \\
\hline Frozen ocean fish* & $0 \cdot 30$ & $0.19,0.41$ & 0.34 & $0 \cdot 31$ & $0 \cdot 28$ & $0 \cdot 17$ & $0 \cdot 21$ \\
\hline Canned ocean fisht & $1 \cdot 4$ & $1 \cdot 1,1 \cdot 7$ & $0 \cdot 70$ & $1 \cdot 4$ & $1 \cdot 8$ & $1 \cdot 5$ & 0.29 \\
\hline Other ocean fish $\ddagger$ & $0 \cdot 82$ & $0 \cdot 40,1 \cdot 2$ & 0.73 & 0.47 & $0 \cdot 37$ & $1 \cdot 8$ & $0 \cdot 01$ \\
\hline Freshwater fish & $2 \cdot 0$ & $1 \cdot 2,2 \cdot 8$ & $2 \cdot 1$ & $1 \cdot 8$ & $2 \cdot 4$ & $1 \cdot 6$ & 0.69 \\
\hline Freshwater predatory fish $\S$ & $1 \cdot 0$ & $0.51,1 \cdot 4$ & $0 \cdot 81$ & $1 \cdot 0$ & $1 \cdot 4$ & 0.72 & $0 \cdot 71$ \\
\hline Vendace & 0.33 & $0.11,0.54$ & 0.65 & $0 \cdot 12$ & $0 \cdot 24$ & $0 \cdot 26$ & 0.44 \\
\hline Other freshwater fishll & 0.69 & $0.37,1.0$ & 0.60 & 0.63 & 0.76 & 0.63 & 0.96 \\
\hline Farmed fish & $1 \cdot 4$ & $1 \cdot 1,1 \cdot 6$ & $1 \cdot 1$ & $1 \cdot 3$ & $1 \cdot 6$ & $1 \cdot 6$ & $0 \cdot 27$ \\
\hline Other fish & 0.02 & $0.00,0.05$ & 0.00 & 0.00 & 0.05 & 0.05 & $0 \cdot 16$ \\
\hline Shellfish & 0.51 & $0.34,0.67$ & $0 \cdot 30$ & 0.50 & 0.32 & $0 \cdot 78$ & 0.01 \\
\hline
\end{tabular}

MeHg, methylmercury.

${ }^{*}$ Coalfish, cod, redfish, fish sticks.

†Tuna, sardine, herring, mackerel.

¥Smoked mackerel, Norwegian salmon.

§Pike, perch, burbot, pike-perch.

IIWhitefish, bream, roach,

-Shrimp, mussel, crab.

Table 5 Adjusted means for consumption of specified fish species (separate health questionnaire, servings/month) across blood MeHg quartiles for women: Fishermen Sub-study, Finland, August 2004-May 2005

\begin{tabular}{|c|c|c|c|c|c|c|c|}
\hline \multirow[b]{3}{*}{ Food groups } & \multicolumn{7}{|c|}{ Adjusted for age and energy intake } \\
\hline & \multicolumn{2}{|c|}{$\begin{array}{l}\text { Total consumption } \\
\text { (servings/month) ( } n \text { 162) }\end{array}$} & \multicolumn{4}{|c|}{$\begin{array}{l}\text { Mean consumption by MeHg quartiles } \\
\text { (servings/month) }\end{array}$} & \multirow{2}{*}{$\begin{array}{l}P \text { for linear } \\
\text { trend }\end{array}$} \\
\hline & Mean & $95 \% \mathrm{Cl}$ & Q1 ( $n$ 42) & Q2 (n 39) & Q3 ( $n$ 40) & Q4 (n 41) & \\
\hline Fish in total & 12 & 11,13 & $6 \cdot 9$ & 11 & 14 & 15 & $<0.001$ \\
\hline Baltic ocean fish & 4.9 & $3 \cdot 9,5 \cdot 9$ & $2 \cdot 7$ & 3.9 & $5 \cdot 6$ & $6 \cdot 7$ & 0.003 \\
\hline Baltic herring & $1 \cdot 6$ & $1 \cdot 2,2 \cdot 0$ & 0.83 & $1 \cdot 3$ & $1 \cdot 6$ & $2 \cdot 3$ & 0.002 \\
\hline Baltic salmon or trout & 0.93 & $0 \cdot 67,1 \cdot 2$ & 0.79 & $1 \cdot 1$ & $0 \cdot 85$ & $1 \cdot 0$ & $0 \cdot 84$ \\
\hline Other Baltic fish & $2 \cdot 4$ & $1 \cdot 7,3 \cdot 1$ & $1 \cdot 1$ & $1 \cdot 5$ & $3 \cdot 2$ & $3 \cdot 5$ & $0 \cdot 01$ \\
\hline Other ocean fish & $2 \cdot 3$ & $1 \cdot 8,2 \cdot 8$ & $1 \cdot 7$ & $2 \cdot 7$ & $2 \cdot 6$ & $2 \cdot 5$ & 0.54 \\
\hline Frozen ocean fish* & 0.45 & $0.35,0.55$ & $0 \cdot 50$ & 0.58 & $0 \cdot 37$ & 0.46 & 0.63 \\
\hline Canned ocean fisht & $1 \cdot 0$ & $0 \cdot 70,1 \cdot 3$ & $0 \cdot 79$ & $1 \cdot 2$ & 0.91 & $1 \cdot 1$ & 0.42 \\
\hline Other ocean fish $\ddagger$ & $0 \cdot 89$ & $0 \cdot 50,1 \cdot 3$ & 0.40 & $1 \cdot 0$ & $1 \cdot 3$ & $0 \cdot 86$ & 0.65 \\
\hline Freshwater fish & $2 \cdot 6$ & $1 \cdot 8,3 \cdot 3$ & $1 \cdot 3$ & $2 \cdot 1$ & $3 \cdot 3$ & $3 \cdot 7$ & 0.03 \\
\hline Freshwater predatory fish $\S$ & $1 \cdot 5$ & $1 \cdot 1,1 \cdot 9$ & 0.52 & $1 \cdot 3$ & $1 \cdot 6$ & $2 \cdot 5$ & 0.007 \\
\hline Vendace & 0.33 & $0.13,0.53$ & $0 \cdot 20$ & 0.06 & $0 \cdot 67$ & 0.42 & 0.33 \\
\hline Other freshwater fishll & $0 \cdot 74$ & $0 \cdot 44,1 \cdot 0$ & 0.55 & $0 \cdot 71$ & $1 \cdot 0$ & $0 \cdot 82$ & $0 \cdot 61$ \\
\hline Farmed fish & $1 \cdot 4$ & $1 \cdot 1,1 \cdot 6$ & 0.86 & $1 \cdot 9$ & $1 \cdot 5$ & $1 \cdot 1$ & $0 \cdot 70$ \\
\hline Other fish & 0.02 & $0.00,0.04$ & 0.01 & 0.01 & 0.04 & 0.01 & $0 \cdot 86$ \\
\hline Shellfish & 0.69 & $0.54,0.84$ & 0.44 & $0 \cdot 80$ & $1 \cdot 0$ & 0.66 & 0.69 \\
\hline
\end{tabular}

MeHg, methylmercury.

${ }^{*}$ Coalfish, cod, redfish, fish sticks.

tTuna, sardine, herring, mackerel.

$\ddagger$ Smoked mackerel, Norwegian salmon.

§ike, perch, burbot, pike-perch.

IIWhitefish, bream, roach,

-Shrimp, mussel, crab.

participants exceeding this level in the present study suggests that in Finland, where domestic Hg emissions are low, exposure to $\mathrm{MeHg}$ may be notable, even when fish consumption is not high. More importantly, fetal exposure to $\mathrm{MeHg}$ may lead to the increased risk of neurotoxic effects also in the general Finnish population. However, owing to the characteristics of the selected study population, the results from the present study do not offer 
adequate grounds to make changes to the current national recommendations on fish consumption, especially since fish is an important source of many beneficial nutrients as well. Therefore, to ensure the health of next generations, $\mathrm{MeHg}$ analysis and exposure assessment should be performed as soon as possible among the general population, especially among women of childbearing age.

Blood MeHg was most strongly associated with fish consumption, as expected. With regard to fish species, Baltic fish seemed to be the most important source of $\mathrm{MeHg}$ for the study population. The strong association is probably explained by the high proportion of Baltic fish in the participants' diet ( $55 \%$ of total fish consumption among men and $41 \%$ among women), since $\mathrm{Hg}$ levels in these Baltic species have generally been low, $0 \cdot 005-0 \cdot 1 \mathrm{mg} / \mathrm{kg}$ fresh weight $(\mathrm{FW})^{(30)}$. The Baltic Sea is the main fishing area for the fishermen and therefore we assume that the high proportion of the fish they consume is from their own catch. To our knowledge, the proportion of Baltic fish among the general Finnish population has not been studied, but it is expected to be lower. $\mathrm{Hg}$ levels in freshwater predatory fish have been significantly higher than in the Baltic species $(0 \cdot 11-0 \cdot 73 \mathrm{mg} / \mathrm{kg} \mathrm{FW})^{(31)}$ and therefore they are often considered as the main source of $\mathrm{Hg}$ for man. Although the consumption of freshwater predatory species in the present study was lower than the consumption of Baltic species, these species were also associated with blood $\mathrm{MeHg}$, especially among women.

Besides fish, elevated concentrations of $\mathrm{Hg}$ have been measured in Finland and other countries from e.g. mushrooms and berries, game meat and offal, vegetables, cereals and drinking water, although the Hg levels measured in these foods have been considerably lower than in fish ${ }^{(32-40)}$. In the present study, the consumption of fruit vegetables, root vegetables, potato, wheat, rye and offal were shown to be positively associated with blood MeHg among one or both sexes but adjustment for fish consumption attenuated the associations for some foods. This probably reflects the fact that these foods are commonly eaten with fish and that fish consumption is a confounding factor in those cases. Nevertheless, the observed associations suggest that in addition to fish, other foods may play an important enough role to be taken into account in future exposure assessments, especially among populations with lower fish consumption than in the present study.

In the present study, some known Hg sources, namely mushrooms and game meats, were not associated with blood MeHg. Since Hg concentrations in these foods may sometimes be as high as in fish ${ }^{(37,38)}$, the absence of an association is probably due to low consumption. Additionally, $\mathrm{Hg}$ in mushrooms exists mostly in the inorganic form, which is absorbed less effectively than MeHg.

Alcoholic beverages, especially wine, were statistically significantly associated with blood MeHg among men.
However, $\mathrm{Hg}$ concentration in wine has been extremely low ${ }^{(41)}$ and it seems unlikely that wine is an important source of $\mathrm{Hg}$. At the same time, alcohol consumption has been shown to potentiate MeHg toxicity and to increase the blood concentration of total $\mathrm{Hg}$ in mice and rats ${ }^{(42,43)}$. Thus, our results suggest that alcohol may interfere with MeHg metabolism by either enhancing the uptake or by impairing its elimination from the body.

For some foods, the association with blood MeHg was observed among men but not among women and vice versa. The differences between the sexes may be partially explained by the considerable differences in diets and nutrient intakes. For example, the consumption of fibre, which may decrease MeHg absorption ${ }^{(28)}$, seemed to be higher among women, possibly explaining why some associations were observed only among men. Additionally, differences in the MeHg excretion and organ distribution between the sexes have been observed in the mouse, rat and $\operatorname{man}^{(44-46)}$. Specifically, males seem to be more effective in eliminating Hg compared with females, which may in part explain the differences in the observed associations between men and women. For example, for game meats, an association was observed among women $(P=0 \cdot 03)$ but not among men $(P=0 \cdot 98)$, even though game consumption was similar among both women and men $(7 \cdot 3$ $(4 \cdot 7-10)$ and $6 \cdot 3(4 \cdot 0-8 \cdot 7) \mathrm{g} / \mathrm{d}$, respectively). However, the underlying reasons for the differences in observed associations between the sexes remain ambiguous.

Validated and accredited methods were used in the present study. Food consumption was measured by a validated FFQ on whole diet. The frequency questions on the consumption of different fish species were not validated, but there was reasonably good correlation between total fish consumption measured by the frequency questions and by the validated FFQ (the age-adjusted correlation coefficients for total fish consumption were 0.64 among men and $0 \cdot 44$ among women ${ }^{(18)}$ ). Blood MeHg concentrations were analysed by an accredited testing laboratory with a method designed for low concentrations. Regarding the limitations of the study, we did not have data on $\mathrm{MeHg}$ concentrations in foods and therefore we could not quantify the relative intake of $\mathrm{MeHg}$ from different foods.

\section{Conclusions}

The present study shows that in addition to fish, other dietary sources such as vegetables, cereals and meat may have a role in $\mathrm{MeHg}$ exposure and they should be taken into account in exposure assessments. The association observed between alcohol consumption and blood MeHg among men is in line with animal studies which have shown that alcohol may potentiate $\mathrm{MeHg}$ accumulation and toxicity and calls for more detailed studies on $\mathrm{MeHg}$ metabolism. Relatively high $\mathrm{MeHg}$ concentrations were observed in the study, and therefore exposure assessment 
should be performed as soon as possible among the general Finnish population, with a special emphasis on women of childbearing age.

\section{Acknowledgements}

This study was funded by the Academy of Finland (decision numbers 77008, 205324, 206950 and 124286). The authors declare they have no conflict of interest. The contributions of each author were as follows: P.K.V. and S.M. were responsible for the concept and design of the study. R.A. and P.R. performed the chemical analysis of samples. R.A. and A.W.T. analysed and interpreted the data and drafted the article. S.M., T.V. and P.K.V. revised the article for important intellectual content. The authors thank the volunteers and research staff of the Fishermen study.

\section{References}

1. Choi AL \& Grandjean P (2008) Methylmercury exposure and health effects in humans. Environ Chem 5, 112-120.

2. Crump KS, Kjellström T, Shipp AM et al. (1998) Influence of prenatal mercury exposure upon scholastic and psychological test performance: benchmark analysis of a New Zealand cohort. Risk Anal 18, 701-713.

3. Davidson PW, Myers GJ, Cox C et al. (2006) Methylmercury and neurodevelopment: longitudinal analysis of the Seychelles Child Development Cohort. Neurotoxicol Teratol 28, 529-535.

4. Debes F, Budtz-Jørgensen E, Weihe P et al. (2006) Impact of prenatal methylmercury exposure on neurobehavioral function at age 14 years. Neurotoxicol Teratol 28, 536-547.

5. Salonen JT, Seppänen K, Nyyssönen K et al. (1995) Intake of mercury from fish, lipid peroxidation, and the risk of myocardial infarction and coronary, cardiovascular, and any death in eastern Finnish men. Circulation 91, 645-655.

6. US Environmental Protection Agency, Integrated Risk Information System (2001) Methylmercury Reference Dose for Chronic Oral Exposure. http://www.epa.gov/iris/subst/ 0073.htm

7. Stern AH \& Smith AE (2003) An assessment of the cord blood:maternal blood methylmercury ratio: implications for risk assessment. Environ Health Perspect 111, 1465-1470.

8. Galal-Gorchev H (1993) Dietary intake, levels in food and estimated intake of lead, cadmium, and mercury. Food Addit Contam 10, 115-128.

9. Chapman L \& Chan HM (2000) The influence of nutrition on methyl mercury intoxication. Environ Health Perspect 108, 29-56.

10. Clarkson TW \& Strain JJ (2003) Nutritional factors may modify the toxic action of methyl mercury in fish-eating populations. J Nutr 133, 5 Suppl. 1, 1539S-1543S.

11. Rice DC (2008) Overview of modifiers of methylmercury neurotoxicity: chemicals, nutrients, and the social environment. Neurotoxicology 29, 761-766.

12. National Research Council, Committee on the Toxicological Effects of Methylmercury \& Board on Environmental Studies and Toxicology (2000) Toxicological Effects of Methylmercury. Washington, DC: National Academy Press; available at http://www.nap.edu/openbook.php?isbn= 0309071402

13. Rice DC (2004) The US EPA reference dose for methylmercury: sources of uncertainty. Environ Res 95, 406-413.
14. Spurgeon A (2006) Prenatal methylmercury exposure and developmental outcomes: review of the evidence and discussion of future directions. Environ Health Perspect 114, 307-312.

15. Turunen AW, Verkasalo PK, Kiviranta $\mathrm{H}$ et al. (2008) Mortality in a cohort with high fish consumption. Int $J$ Epidemiol 37, 1008-1017.

16. Männisto S, Virtanen M, Mikkonen $\mathrm{T}$ et al. (1996) Reproducibility and validity of a food frequency questionnaire in a case-control study on breast cancer. J Clin Epidemiol 49, 401-409.

17. Paalanen L, Männisto S, Virtanen MJ et al. (2006) Validity of a food frequency questionnaire varied by age and body mass index. J Clin Epidemiol 59, 994-1001.

18. Turunen AW, Männistö S, Kiviranta H et al. (2010) Dioxins, polychlorinated biphenyls, methyl mercury and omega-3 polyunsaturated fatty acids as biomarkers of fish consumption. Eur J Clin Nutr 64, 313-323.

19. National Public Health Institute, Nutrition Unit (2005) Fineli. Finnish Food Composition Database, Release 5. Helsinki: National Public Health Institute; available at http://www.fineli.fi/

20. Baxter DC, Rodushkin I, Engström E et al. (2007) Methylmercury measurement in whole blood by isotopedilution GC-ICPMS with 2 sample preparation methods. Clin Chem 53, 111-116.

21. Yan J (2001) Isotope Pattern Calculator v4.5. http:// yanjunhua.tripod.com/pattern1.htm

22. Yang L, Colombini V, Maxwell P et al. (2003) Application of isotope dilution to the determination of methylmercury in fish tissue by solid-phase microextraction gas chromatography-mass spectrometry. J Chromatogr A 1011, 135-142.

23. Paturi M, Tapanainen H, Reinivuo H et al. (editors) (2008) The National FINDIET 2007 Survey. Publication of the National Public Health Institute no. B23/2008. Helsinki: National Public Health Institute.

24. Svensson BG, Nilsson A, Jonsson E et al. (1995) Fish consumption and exposure to persistent organochlorine compounds, mercury, selenium and methylamines among Swedish fishermen. Scand J Work Environ Health 21, 96-105.

25. Cole DC, Kearney J, Sanin LH et al. (2004) Blood mercury levels among Ontario anglers and sport-fish eaters. Environ Res 95, 305-314.

26. Hsu CS, Liu PL, Chien LC et al. (2007) Mercury concentration and fish consumption in Taiwanese pregnant women. BJOG 114, 81-85.

27. Berglund $\mathrm{M}$, Lind $\mathrm{B}$, Björnberg KA et al. (2005) Interindividual variations of human mercury exposure biomarkers: a cross-sectional assessment. Environ Health Global Access Sci Source 4, 20.

28. Rowland IR, Mallett AK, Flynn J et al. (1986) The effect of various dietary fibres on tissue concentration and chemical form of mercury after methylmercury exposure in mice. Arch Toxicol 59, 94-98.

29. Landry TD, Doherty RA \& Gates AH (1979) Effects of three diets on mercury excretion after methylmercury administration. Bull Environ Contam Toxicol 22, 151-158.

30. Venäläinen E-R, Hallikainen A, Parmanne R et al. (2004) Heavy Metal Contents in Finnish Sea and Fresh Water Fish. National Food Agency Publication no. 3/2004. Helsinki: National Food Agency.

31. Munthe J, Wängberg I, Rognerud S et al. (2007) Mercury in Nordic Ecosystems. IVL Report no. B1761. Gothenburg: IVL Swedish Environmental Research Institute Ltd.

32. Larsen EH, Andersen NL, Møller A et al. (2002) Monitoring the content and intake of trace elements from food in Denmark. Food Addit Contam 19, 33-46.

33. Ysart G, Miller P, Croasdale M et al. (2000) 1997 UK Total Diet Study - dietary exposures to aluminium, arsenic, cadmium, chromium, copper, lead, mercury, nickel, selenium, tin and zinc. Food Addit Contam 17, 775-786. 
34. Lindberg A, Björnberg KA, Vahter M et al. (2004) Exposure to methylmercury in non-fish-eating people in Sweden. Environ Res 96, 28-33.

35. Rubio C, Gutiérrez A, Burgos A et al. (2008) Total dietary intake of mercury in the Canary Islands, Spain. Food Addit Contam 25, 946-952.

36. Sirot V, Guérin T, Mauras Y et al. (2008) Methylmercury exposure assessment using dietary and biomarker data among frequent seafood consumers in France CALIPSO study. Environ Res 107, 30-38.

37. Fischer RG, Rapsomanikis S, Andreae MO et al. (1995) Bioaccumulation of methylmercury and transformation of inorganic mercury by macrofungi. Environ Sci Technol 29, 993-999.

38. Falandysz J \& Gucia M (2008) Bioconcentration factors of mercury by parasol mushroom (Macrolepiota procera). Environ Geochem Health 30, 121-125.

39. Liukkonen-Lilja H (1993) Mercury. Pollutant Database I. National Food Administration Research Notes no. 1/1993. Helsinki: National Food Administration.

40. Mustaniemi A, Hallikainen A \& Witick A (1994) Mercury Intake from Fish and Other Food. National Food Administration
Research Notes no. 13/1994. Helsinki: National Food Administration.

41. Catarino S, Curvelo-Garcia AS \& de Sousa RB (2008) Revisão: elementos contaminantes nos vinhos. Ciência Téc Vitiv 23, 3-19.

42. Rumbeiha WK, Gentry PA \& Bhatnagar MK (1992) The effects of administering methylmercury in combination with ethanol in the rat. Vet Hum Toxicol 34, 21-25.

43. Turner CJ, Bhatnagar MK \& Yamashiro S (1981) Ethanol potentiation of methyl mercury toxicity: a preliminary report. J Toxicol Environ Health 7, 665-668.

44. Nielsen JB (1992) Toxicokinetics of mercuric-chloride and methylmercuric chloride in mice. J Toxicol Environ Health 37, 85-122.

45. Magos L, Peristianis GC, Clarkson TW et al. (1981) Comparative-study of the sensitivity of male and female rats to methylmercury. Arch Toxicol 48, 11-20.

46. Thomas DJ, Fisher HL, Sumler MR et al. (1986) Sexual differences in the distribution and retention of organic and inorganic mercury in methyl mercury-treated rats. Environ Res 41, 219-234. 